\title{
ESTRATEGIAS PARA MEJORAR EL POSICIONAMIENTO DE LA MARCA PERÚ EN EL MERCADO ITALIANO
}

\section{STRATEGIES TO IMPROVE THE POSITIONING OF THE PERU BRAND IN THE ITALIAN MARKET}

\author{
Neptali Barnett Valdivia ${ }^{1}$
}

\author{
Palabras clave: \\ estrategias, valor \\ agregado, \\ posicionamiento \\ de marca, marca \\ país.
}

\begin{abstract}
Resumen
La Marca Perú, tiene un lugar ganado en el mercado interno e internacional, pero no es suficiente; será mayor en la medida que se desarrollen estrategias de posicionamiento que permitan la colocación de los productos que exportemos; pero será, en todo caso, productos con valor agregado, puesto que formar parte de una economía primaria, de producción de materia prima e insumos, genera que otros se beneficien en la transformación de dichos productos en bienes acabados, en la que poseen mejores precios, y lo que es más valioso, permitan la generación de mayores puestos de trabajo; de esto nos estamos perdiendo y quedando en la condición de país productor de materia. El objetivo de esta investigación fue analizar las medidas de colocación de los productos de origen nacional con valor agregado en el mercado italiano para mejorar la balanza comercial, permitiendo superar las dificultades que tradicionalmente limitaban las posibilidades del desarrollo económico social del país. Se realizó una investigación de tipo cuantitativa y transeccional, con una muestra de 80 participantes: 43 funcionarios de PROMPERÚ y 37 funcionarios de las empresas exportadoras hacia el mercado italiano. Este estudio contribuye con nuevas estrategias de posicionamiento de la Marca Perú basadas en las características del producto, calidad, precio, uso, calidad de vida de los usuarios; aumentando la competitividad de los productos peruanos con valor agregado en el mercado italiano. Entre estas estrategias se destaca la de "posicionamiento por beneficios" resaltando los principales beneficios del producto, siendo una tendencia de selección entre los consumidores finales italianos a la hora de consumir los productos de la Marca Perú. Se concluye que existe una influencia significativa de las estrategias de posicionamiento de los productos de la Marca Perú en el mercado italiano para lograr mayores niveles de exportación de producto con valor agregado.
\end{abstract}

Códigos JEL: M30, M31

\footnotetext{
${ }^{1}$ Universidad de Lima, (Perú).

E-mail: nbarnett@ulima.edu.pe
} 
Keywords:

strategies, added

value, brand

positioning,

country brand

\begin{abstract}
The Peru Brand has a place earned in the domestic and international market, but it is not enough; It will be greater to the extent that positioning strategies are developed that allow the placement of the products that we export; but it will, in any case, be products with added value, since being part of a primary economy, producing raw materials and inputs, generates that others benefit in the transformation of said products into finished goods, in which they have better prices, and what is more valuable, generating more jobs; Of this we are losing ourselves and remaining in the condition of a country that produces material. The objective of this research was to analyze the measures of placement of products of national origin with added value in the Italian market to improve the trade balance, to allow overcoming the difficulties that traditionally limited the possibilities of the economic and social development of the country. A quantitative and transectional investigation was carried out, with a sample of 80 participants: 43 officials from PROMPERÚ and 37 officials from exporting companies to the Italian market. This study contributed with new positioning strategies for the Peru Brand based on the characteristics of the product, quality, price, use, quality of life of the users; Increases the competitiveness of Peruvian products with added value in the Italian market. Among these strategies, the one of "positioning by benefits" stands out, highlighting the main benefits of the product, being a selection trend among Italian final consumers when consuming the Peru Brand products. It is concluded that there is a significant influence of the positioning strategies of the Peru Brand products in the Italian market to achieve higher levels of export of value-added products.
\end{abstract}

\section{INTRODUCCIÓN}

En la actualidad los países tienden a formar bloques económicos porque sus recursos naturales no son homogéneos sino diversos, del mismo modo los medios de producción tienden a especializarse en el desarrollo de tecnologías productivas. Existe la necesidad de tomar medidas estratégicas en la colocación de productos en el mercado internacional, los cuales no pueden poseer las mismas ventajas actuando unilateralmente, confiando en sus propios potenciales productivos. Así vemos que aún, los países industrializados del primer mundo, con una tecnología productiva de punta, precisan realizar alianzas, bloques y convenios con otros para proveerse de recursos que por sí mismos no poseen.
Hay aspectos de carácter político-social que facilitan la colocación de un producto en el mercado internacional, como son las normas internacionales y nacionales que viabilizan su operatividad, convenios, constitución de bloques, estrategias de posicionamiento y procedimientos de diversa índole para hacer que dicho producto sea el preferido entre los consumidores (Pérez et al 2018; Pino et al, 2016).

Perú es un país exportador, fundamentalmente de materias primas e insumos (Orihuela y Echenique, 2019), con muy pocos productos con valor agregado (Tosoni, 2017; Schwarz, Mathijs, y Maertens, 2019); la figura a continuación (Figura 1) muestra la importancia que cobra para el país la actividad exportadora, así como su significado para el desarrollo social, siendo sus principales destinos China (26,3\%), 
Estados Unidos $(15,6 \%)$ y la Unión Europea $(14,7 \%)$.

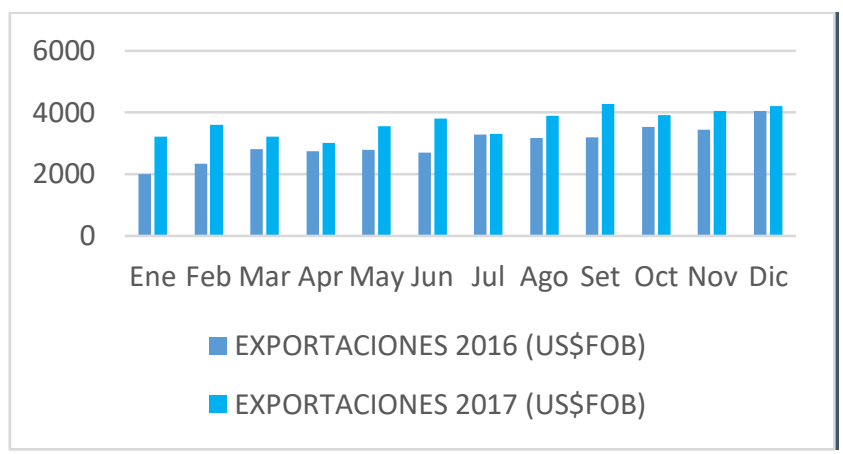

Figura 1. Exportaciones del Perú 2016-2017 en millones de dólares.

Fuente: Adex Data Trade. Elaboracion propia.

La Marca Perú debe caracterizar a todos los peruanos, fundamentalmente en otros países a los que se quiere llegar con los productos más significativos de la nación (Bassols, 2016). Es necesario destacar la identidad competitiva para mostrar el potencial turístico, actividades de exportación e inversiones, impulsando así el empleo (Ávalo, Yagüe y Cangahuala, 2016). La empresa (o el país), deberá poseer un prestigio ganado por la calidad de sus productos, que se logra con las estrategias de posicionamiento elaborada por los promotores de dicha marca, para que ésta se haga permanente en la conciencia de los consumidores (Grohs et al, 2016; Niembro, 2017). La imagen del país puede funcionar como una variable de resumen de la información y los atributos del producto de un país en particular (Bruwer et al., 2014). La marca es exitosa en Perú porque ha reunido un grupo estratégico de ideas culturales positivas respecto al Perú y la peruanidad (Silverman, 2015). Dichas estrategias considerarán las exportaciones en los sectores químico, metal mecánico y agropecuario, al que se suman las manufacturas orientadas en una política de integración comercial, aprovechando la infraestructura instalada para la exportación (Vázquez y Morales, 2017). El posicionamiento de la Marca Perú está con relación a las características de sus productos, sustentadas en calidad, precio, uso y aplicación.

Se reconoce que hay otros países que han optado por acciones que repercuten en el posicionamiento en la exportación de su marca país, provocando consecuencias en sus actividades económicas y sociales. En la opinión de Felipa (2014) entre esas estrategias se pueden mencionar las siguientes:

- Fortalecimiento de la institucionalidad del país, lo que incluye factores como derechos de propiedad, solución de conflictos para mejorar el clima de negocios del país a través de políticas económicas apropiadas, tributarias y laborales, estabilidad política, estabilidad económica y social, además mejorar el acceso a los recursos.

- Reducción de las brechas de infraestructura física (transporte) y la provisión de los servicios relacionados para la integración de mercados y el desarrollo empresarial.

- Fomento de innovación tecnológica e inversión para el aumento de la aplicación de conocimiento con el objetivo de mejorar la competitividad de la producción usando las herramientas que provee la ciencia.

- $\quad$ Simplificación de las regulaciones para los negocios, como leyes laborales, pago de impuestos, cierre de empresas y permisos de construcción.

Esta investigación tiene como objetivo analizar las estrategias que puedan lograr un posicionamiento mayor a través de la Marca Perú, de tal manera que puedan elevarse las exportaciones de productos con valor agregado, eligiendo para tal efecto el mercado italiano.

\section{Exportaciones a Italia}

La siguiente tabla (Tabla 1) ilustra las posibilidades de desarrollar políticas de exportación en virtud al Tratado de Libre Comercio (TLC) firmado con Italia, de estos datos se puede inferir las acciones que permitan elaborar una estrategia de posicionamiento para acceder a dicho mercado, poniendo énfasis en los productos con valor agregado. 
Tabla 1

Indicadores macroeconómicos 2015: Italia Perú

\begin{tabular}{|c|c|c|}
\hline & Italia & Perú \\
\hline Población (Millones) & 60,2 & 31,2 \\
\hline PBI (US\$ Millones) & 1842835 & 212700 \\
\hline PBI per cápita (US\$) & 30594 & 6828 \\
\hline Exportación Bs. y Ss. (\% PBI) ${ }^{1}$ & $30 \%$ & $22 \%$ \\
\hline Importación Bs. y Ss. (\% PBI) ${ }^{1}$ & $28 \%$ & $24 \%$ \\
\hline $\begin{array}{l}\text { Intercambio Comercial Bs. y } \\
\text { Ss. }(\% \mathrm{PBI})^{1}\end{array}$ & $58 \%$ & $46 \%$ \\
\hline $\begin{array}{l}\text { IED: Flujo Neto (US\$ } \\
\text { Millones)1 }\end{array}$ & 19091 & 7607 \\
\hline $\begin{array}{l}\text { Tipo de Cambio Promedio } \\
\text { (Moneda Nacional / US\$) }^{1}\end{array}$ & 0,8 & 2,8 \\
\hline
\end{tabular}

Fuente: WEO, EUROSTAT, OCDE, BCRP, INEI Elaboración: MINCETUR-OGEE-OEEI.

1Información al 2014, debido a que no se dispone de proyecciones al 2015 para el socio comercial.

Es importante mencionar que las importaciones mantenidas con Italia han sido mayores que las exportaciones entre el 2013 y 2017, PROMPERÚ (2018).

\section{Exportaciones en los sectores químico, metalmecánica y agropecuario}

Las exportaciones no tradicionales representan el $30 \%$ de las exportaciones constituidas por el sector agropecuario y químico. De acuerdo con cifras de la Sunat, en el periodo enero-octubre de 2018, las exportaciones peruanas del sector químico alcanzaron un valor de US\$ 1,281 millones, un $13.5 \%$ más que en el mismo periodo del año anterior.

Respecto de los productos exportados en el periodo analizado, el principal fue las demás películas de polímeros de propileno, cuyos envíos ascendieron a US\$ 100 millones, a este le siguieron el óxido de zinc (US\$ 97 millones; $+32.7 \%$ ), las demás películas de polímeros de etileno (US\$ 59 millones; $+25.6 \%$ ), el ácido sulfúrico (US\$ 55 millones; +171\%) y las lacas colorantes (US\$ 54 millones; +11\%).
En el periodo analizado, el aumento en las exportaciones del sector se habría dado tanto por el incremento en la demanda de nuestros principales compradores, como por la mayor penetración en mercados con poca presencia de productos peruanos de este sector.

Según cifras del Centro de Comercio Internacional, los principales importadores de las demás películas de polímeros de propileno a nivel mundial en 2017 fueron EE.UU. (con un 9\% del total), Alemania (7\%), China (6\%), Francia (5\%) y Reino Unido (5\%). Cabe mencionar que los principales proveedores a nivel mundial de este producto en 2017 fueron Alemania, Italia y China, con una participación del $11.9 \%, 8.8 \%$ y $6.3 \%$, respectivamente, mientras que Perú obtuvo solo un $1.1 \%$.

Resulta clave impulsar políticas que ayuden a los exportadores peruanos a penetrar nuevos mercados y establecer un marco legal que incentive las inversiones y la formación de capital humano altamente calificado, son algunas de las políticas claves para potenciar la competitividad de este y otros sectores (Curzi et al, 2020; Lámbarry, 2016).

\section{Políticas de integración comercial}

La constitución de la Unión Europea como un bloque comercial, permitió según el establecimiento de sus propias normas, la firma de un TLC con cualquier país europeo (incluyendo Italia), pero sujeta a las normas colectivas establecidas para cualquier tipo de transacciones comerciales.

Las relaciones comerciales del Perú con Italia están comprendidas dentro de esta normativa; no es un trato bilateral, sino del Perú con la Unión Europea, que tiene entre sus miembros a Italia. Las disciplinas incluidas en la negociación fueron: "Acceso a Mercados; Reglas de Origen; Asuntos Aduaneros y Facilitación del Comercio; Obstáculos Técnicos al Comercio; Medidas Sanitarias y Fitosanitarias; Defensa Comercial; Servicios, Establecimiento y Movimiento de Capitales; Compras Públicas; Propiedad Intelectual; Competencia; Solución de Diferencias, Asuntos Horizontales e 
Institucionales; Comercio y Desarrollo Sostenible y Asistencia Técnica y Fortalecimiento de Capacidades". (MINCETUR, 2017).

\section{METODOLOGÍA}

Se realizó una investigación cuantitativa, transeccional, de diseño simple no experimental. La muestra la constituyen 80 participantes: 43 funcionarios de PROMPERÚ y 37 funcionarios de las empresas exportadoras hacia el mercado italiano que permitirán la recogida de los datos específicos realizados sobre las exportaciones con valor agregado a la República de Italia. Para la recolección de datos se utilizaron técnicas de observación, encuesta y entrevista.

\section{Análisis inferencial}

Por ser una investigación paramétrica, en el contraste de las hipótesis se utilizó el estadístico $Z$, dado que la muestra empleada es mayor a 31 participantes, de acuerdo a la siguiente fórmula:

$$
Z=\frac{\bar{X}_{1}-\bar{X}_{2}}{\sqrt{\frac{\sigma_{1}^{2}}{n_{1}}+\frac{\sigma_{2}^{2}}{n_{2}}}}
$$

\section{Hipótesis Específica 1}

Con un nivel de significancia del $0.05 \%$ se desea saber si las estrategias de posicionamiento basadas en las características de los productos Marca Perú en el mercado italiano tuvo un mejoramiento en las exportaciones de productos con valor agregado en los sectores químico, metal mecánico y agropecuario.

Datos:

Estrategias de posicionamiento basadas en las características de productos Marca Perú en el mercado italiano.

$\mathrm{n} 1=43$

$\bar{X}_{1}=20.65$

$\sigma 1=2.78$

Productos con valor agregado en los sectores químico, metalmecánico y agropecuario. $\mathrm{n} 2=37$

$\bar{X}_{2}=22.94$

$\sigma 2=2.43$

a) Ho: $\mu 1=\mu 2$

No existen diferencias significativas en las estrategias de posicionamiento basadas en las características de los productos Marca Perú en el mercado italiano para el mejoramiento de las exportaciones de productos con valor agregado en los sectores químico, metalmecánico y agropecuario.

b) Ha: $\mu 1>\mu 2$

Existen diferencias significativas en las estrategias de posicionamiento basadas en las características de los productos Marca Perú en el mercado italiano para el mejoramiento de las exportaciones de productos con valor agregado en los sectores químico, metalmecánico y agropecuario.

$$
Z=\frac{\bar{X}_{1}-\bar{X}_{2}}{\sqrt{\frac{\sigma_{1}^{2}}{n_{1}}+\frac{\sigma_{2}^{2}}{n_{2}}}}=\frac{20.65-22.94}{\sqrt{\frac{7.73}{43}+\frac{5.9}{37}}}=\frac{-2.29}{\sqrt{0.18+0.16}}=\frac{-2.29}{\sqrt{0.34}}=\frac{-2.29}{0.58}=-\mathbf{3 . 9 5}
$$

\section{$3.95>1.645$}

\section{Hipótesis Específica 2}

Con un nivel de significancia del $0.05 \%$ se desea saber si las estrategias de posicionamiento basadas en la calidad y precio de los productos Marca Perú en el mercado italiano tuvo un mejoramiento en las exportaciones de productos con valor agregado en el sector manufacturero.

Datos:

Estrategias de posicionamiento basadas en la calidad y precio de productos Marca Perú en el mercado italiano.

$\mathrm{n}_{1}=43$

$\bar{X}_{1}=21.53$

$\sigma_{1}=2.67$

Productos con valor agregado en el sector manufactura.

$\mathrm{n} 2=37$

$\bar{X}_{2}=23.08$

$\boldsymbol{\sigma}_{2}=2.67$ 
a) Ho: $\mu 1=\mu 2$

No existen diferencias significativas en las estrategias de posicionamiento de los productos Marca Perú en el mercado italiano basadas en la calidad y precio sobre el mejoramiento de las exportaciones de productos con valor agregado en el sector manufacturero.

b) $\mathrm{H}_{\mathrm{a}}: \mu_{1}>\mu_{2}$

Existen diferencias significativas en las estrategias de posicionamiento de los productos Marca Perú en el mercado italiano basadas en la calidad y precio sobre el mejoramiento de las exportaciones de productos con valor agregado en el sector manufacturero.

$$
Z=\frac{\bar{X}_{1}-\bar{X}_{2}}{\sqrt{\frac{\sigma_{1}^{2}}{n_{1}}+\frac{\sigma_{2}^{2}}{n_{2}}}}=\frac{21.53-23.08}{\sqrt{\frac{7.13}{43}+\frac{7.13}{37}}}=\frac{-1.55}{\sqrt{\mathbf{0 . 1 7 + 0 . 1 9}}}=\frac{-1.55}{\sqrt{\mathbf{0 . 3 6}}}=\frac{-1.55}{0.6}=-\mathbf{2 . 5 8}
$$$$
-2.58>1.645
$$

\section{Hipótesis Específica 3}

Con un nivel de significancia del $0.05 \%$ se desea saber si las estrategias de posicionamiento basadas en el uso y aplicación de los productos Marca Perú en el mercado italiano tuvo un mejoramiento en las exportaciones de productos con valor agregado sustentadas en políticas de integración comercial.

Datos:

Estrategias de posicionamiento basadas en el uso y aplicación de productos Marca Perú en el mercado italiano.

$\mathrm{n}_{1}=43$

$\bar{X}_{1}=21.09$

$\boldsymbol{\sigma}_{1}=2.41$

Productos con valor agregado sustentadas en políticas de integración comercial.

$\mathrm{n}_{2}=37$

$$
\bar{X}_{2}=22.65
$$

$\sigma_{2}=4.55$

$$
\text { a) } H_{0}: \mu_{1}=\mu_{2}
$$

No existen diferencias significativas en las estrategias de posicionamiento basadas en el uso y aplicación de los productos Marca Perú en el mercado italiano sobre el mejoramiento de las exportaciones de productos con valor agregado sustentadas en políticas de integración comercial.

b) $\mathrm{H}_{\mathrm{a}}: \mu_{1} \neq \mu_{2}$

Existen diferencias significativas en las estrategias de posicionamiento basadas en el uso y aplicación de los productos Marca Perú en el mercado italiano sobre el mejoramiento de las exportaciones de productos con valor agregado sustentadas en políticas de integración comercial.

$$
Z=\frac{\bar{X}_{1}-\bar{X}_{2}}{\sqrt{\frac{\sigma_{1}^{2}}{n_{1}}+\frac{\sigma_{2}^{2}}{n_{2}}}}=\frac{21.09-22.65}{\sqrt{\frac{5.81}{43}+\frac{20.7}{37}}}=\frac{-1.56}{\sqrt{0.13+0.56}}=\frac{-1.56}{\sqrt{0.69}}=\frac{-1.56}{0.83}=-1.87
$$

\section{$1.87>1.645$}

\section{Hipótesis Específica 4}

Con un nivel de significancia del $0.05 \%$ se desea saber si las estrategias de posicionamiento basadas en el estilo de vida de los consumidores de los productos Marca Perú en el mercado italiano tuvo un mejoramiento en las exportaciones de productos con valor agregado al poseer una infraestructura moderna para la exportación.

Datos:

Estrategias de posicionamiento basadas en el estilo de vida de los consumidores de productos Marca Perú en el mercado italiano.

$\mathrm{n}_{1}=43$

$\bar{X}_{1}=20.81$

$\sigma_{1}=3.13$

Productos con valor agregado al poseer una infraestructura instalada para la exportación

$$
\begin{aligned}
& \mathrm{n}_{2}=37 \\
& \bar{X}_{2}=22.27 \\
& \sigma_{2}=3.17
\end{aligned}
$$

\section{a) $\mathrm{H}_{\mathrm{o}}: \mu_{1}=\mu_{2}$}

No existen diferencias significativas en las estrategias de posicionamiento basadas en el estilo de vida de los consumidores de los productos Marca Perú en el mercado italiano para el mejoramiento de las exportaciones de productos con valor agregado al poseer una 
moderna infraestructura instalada para la exportación.

\section{c) $\mathrm{H}_{\mathrm{a}}: \mu_{1} \neq \mu_{2}$}

Existen diferencias significativas en las estrategias de posicionamiento basadas en el estilo de vida de los consumidores de los productos Marca Perú en el mercado italiano para el mejoramiento de las exportaciones de productos con valor agregado al poseer una moderna infraestructura instalada para la exportación.

$Z=\frac{\bar{X}_{1}-\bar{X}_{2}}{\sqrt{\frac{\sigma_{1}^{2}}{n_{1}}+\frac{\sigma_{2}^{2}}{n_{2}}}}=\frac{20.81-22.27}{\sqrt{\frac{9.8}{43}+\frac{10}{37}}}=\frac{-1.46}{\sqrt{0.23+0.27}}=\frac{-1.46}{\sqrt{0.5}}=\frac{-1.46}{0.7}=-\mathbf{2 . 0 8}$ $-2.08>1.645$

\section{Hipótesis Específica 5}

Con un nivel de significancia del $0.05 \%$ se desea saber si las estrategias de posicionamiento basada en la competencia de los productos Marca Perú en el mercado italiano tuvo un mejoramiento en las exportaciones de productos con valor agregado generando mayores puestos de trabajo.

\section{Datos:}

Estrategias de posicionamiento basadas en la competencia de los productos Marca Perú en el mercado italiano.

$\mathrm{n}_{1}=43$

$\bar{X}_{1}=20.97$

$\sigma_{1}=2.3$

Productos con valor agregado generando mayores puestos de trabajo.

$\mathrm{n} 2=37$

$\bar{X}_{2}=23.13$

$\sigma 2=2.98$

$$
\text { a) } \mathrm{H}_{\mathrm{o}}: \mu_{1}=\mu_{2}
$$

No existen diferencias significativas en las estrategias de posicionamiento basadas en la competencia de los productos Marca Perú en el mercado italiano para el mejoramiento de productos con valor agregado generando mayores puestos de trabajo.
b) $\mathrm{H}_{\mathrm{a}}: \mu_{1} \neq \mu_{2}$

Existen diferencias significativas en las estrategias de posicionamiento basadas en la competencia de los productos Marca Perú en el mercado italiano para el mejoramiento de productos con valor agregado generando mayores puestos de trabajo.

$Z=\frac{\bar{X}_{1}-\bar{X}_{2}}{\sqrt{\frac{\sigma_{1}^{2}}{n_{1}}+\frac{\sigma_{2}^{2}}{n_{2}}}}=\frac{20.97-23.13}{\sqrt{\frac{5.29}{43}+\frac{8.88}{37}}}=\frac{-2.16}{\sqrt{0.12+0.24}}=\frac{-2.16}{\sqrt{0.36}}=\frac{-2.16}{0.6}=-3.6$

$-3.6>1.645$

\section{Hipótesis Principal}

Con un nivel de significancia del $0.05 \%$ se desea saber si las estrategias de posicionamiento de los productos Marca Perú en el mercado italiano tuvo un mejoramiento en las exportaciones de productos con valor agregado.

\section{Datos:}

Estrategias de posicionamiento de los productos Marca Perú en el mercado italiano. $\mathrm{n}_{1}=43$

$\bar{X}_{1}=105.07$

$\boldsymbol{\sigma}_{1}=13.41$

Exportación de productos con valor agregado. $\mathbf{n}_{2}=37$

$\bar{X}_{2}=114.08$

$\boldsymbol{\sigma}_{2}=23.15$

$$
\text { a) } H_{0}: \mu_{1}=\mu_{2}
$$

No existen diferencias significativas en las estrategias de posicionamiento de los productos Marca Perú en el mercado italiano para el mejoramiento de las exportaciones de los productos con valor agregado.

$$
\text { b) } H_{a}: \mu_{1} \neq \mu_{2}
$$

Existen diferencias significativas en las estrategias de posicionamiento de los productos Marca Perú en el mercado italiano para el mejoramiento de las exportaciones de los productos con valor agregado. 
$Z=\frac{\bar{X}_{1}-\bar{X}_{2}}{\sqrt{\frac{\sigma_{1}^{2}}{n_{1}}+\frac{\sigma_{2}^{2}}{n_{2}}}}=\frac{105.07-114.08}{\sqrt{\frac{179.83}{43}+\frac{535.92}{37}}}=\frac{-9.01}{\sqrt{4.18+14.48}}=\frac{-9.01}{\sqrt{18.66}}=\frac{-9.01}{4.32}=-\mathbf{2 . 0 8}$

$-2.08>1.645$

\section{RESULTADOS}

Promedios sobre las estrategias de posicionamiento basadas en las características de productos Marca Perú en el mercado italiano para el mejoramiento de las exportaciones de productos con valor agregado en los sectores químico, metalmecánico y agropecuari

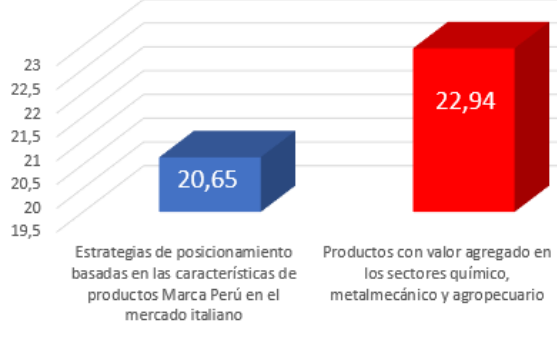

Figura 2.

Fuente: Adex Data Trade

Promedios sobre las estrategias de posicionamiento basadas en la calidad y precio de los productos Marca Perú en el mercado italiano para el mejoramiento de las exportaciones de productos con valor agregado en el sector manufactura.

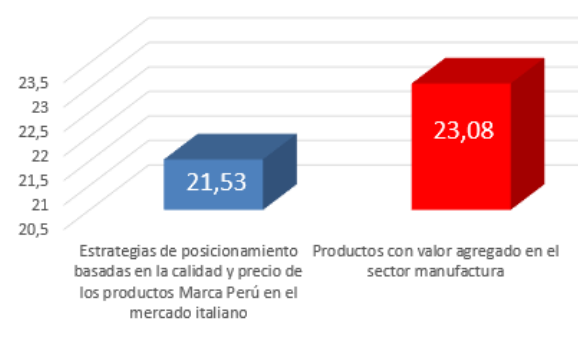

Figura 3.

Fuente: Adex Data Trade

Promedios sobre las estrategias de posicionamiento basadas en e uso y aplicaciones de los productos Marca Perú en el mercado italiano para el mejoramiento de las exportaciones de productos con valor agregado sustentadas en políticas de integración comerc

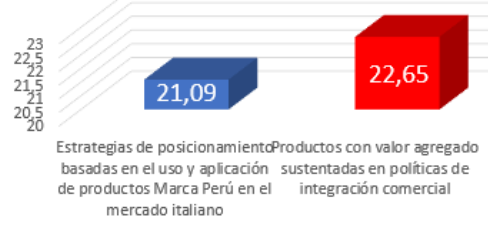

Figura 4.

Fuente: Adex Data Trade
Promedios sobre las estrategias de posicionamiento basadas en el estilo de vida de los consumidores de los productos Marca Perú en el mercado italiano para el mejoramiento de las exportaciones de productos con valor agregado al poseer una infraestructura

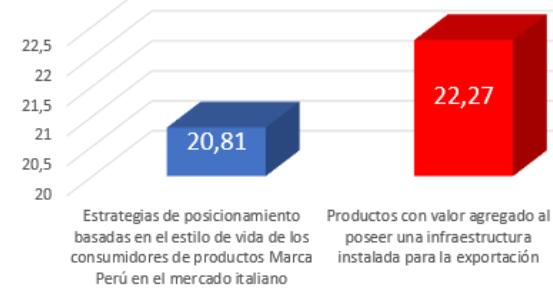

Figura 5.

Fuente: Adex Data Trade

Promedios sobre las estrategias de posicionamiento basadas en la competencia de los productos Marca Perú en el mercado italiano para el mejoramiento de las exportaciones de productos con valor agregado generando mayores puestos de trabajo

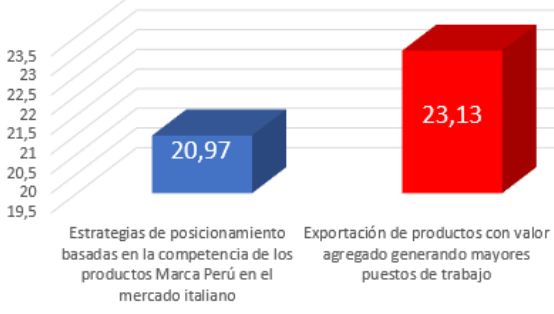

Figura 6.

Fuente: Adex Data Trade

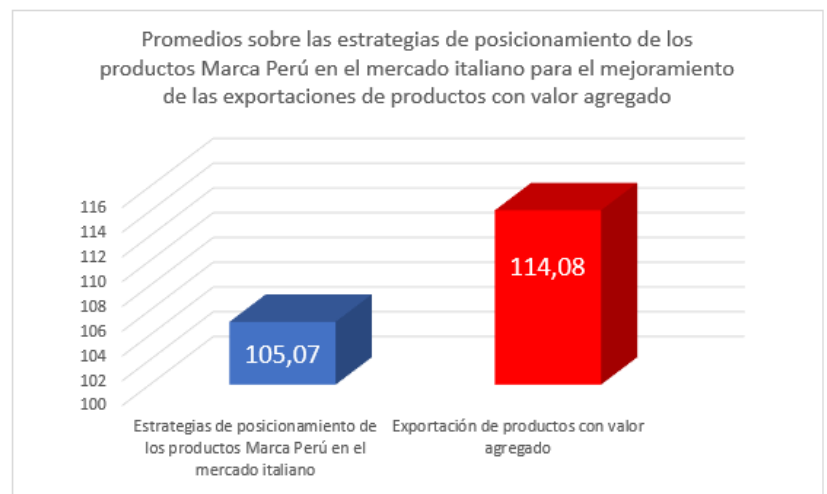

Figura 7.

Fuente: Adex Data Trade 


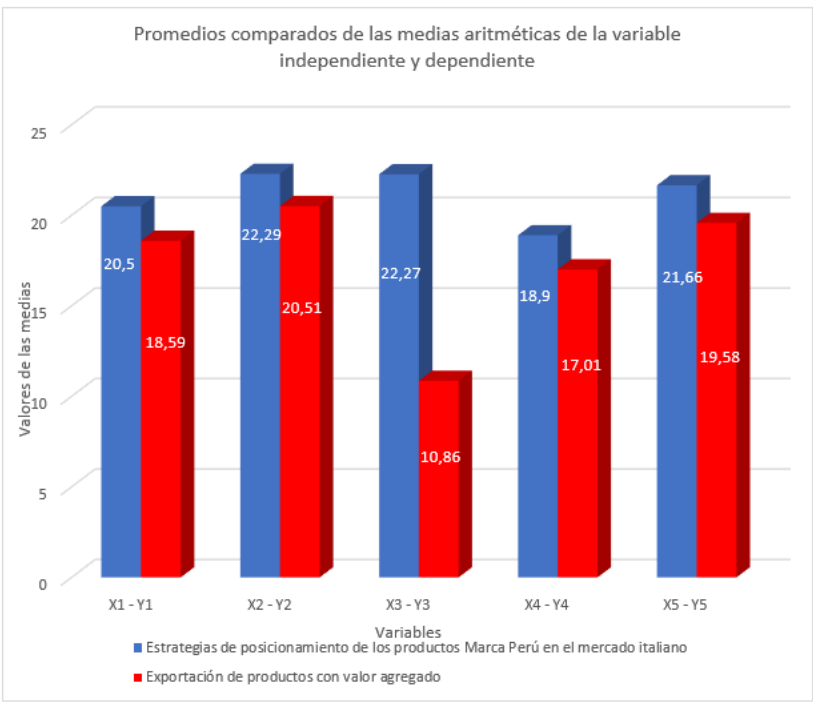

Figura 8.

Fuente: Adex Data Trade

\section{DISCUSIÓN}

En esta etapa de la globalización de la economía, los profesionales de las ciencias administrativas y de las ciencias económicas, escudriñan el mercado internacional para inferir de ellas la dinámica que mueve el desarrollo de las naciones; es imposible concebir dicho desarrollo encerrada en las fronteras físicas de cada país, por ello deberá tomar en cuenta los factores y las condiciones de dicho desarrollo en la multitud de interrelaciones en que las naciones establecen entre sí, siendo una de ellas las relaciones comerciales.

Existe una relación significativa entre las estrategias de posicionamiento de la Marca Perú en el mercado italiano y el incremento de las exportaciones de productos con valor agregado; desarrollando estas preferencias debido a las campañas como parte de las estrategias que desarrollaran las instituciones encargadas con este objetivo, teniendo sustento las que se refieren a la calidad intrínseca del producto, que le dan valor específico a los productos como productos acabados con grados de aceptación en los consumidores de otros mercados y de Italia.

En relación con los logros propuestos en la hipótesis específica 1, que propone el desarrollo de estrategias de la Marca Perú con relación a las características del producto, sustentado en su fácil manejo, durabilidad, conservación e insumos y materiales del producto, se posibilita las exportaciones en los sectores químico, metalmecánico y agropecuario, manteniendo incrementos significativos en cuanto al sector metalmecánico, teniendo signo negativo en los productos agropecuarios. Este resultado se contrapone con el estudio realizado por de Azambuja et al (2019), basado en los resultados empíricos el cual señala que cuanto mejor sea la imagen país del Perú, mayor será la intención de comprar un producto agropecuario por parte de un consumidor extranjero.

En la hipótesis específica 2, referido al desarrollo de las estrategias de posicionamiento de la Marca Perú basadas en la calidad y precio de los productos Marca Perú que miran su funcionalidad, versatilidad, comodidad, seguridad y accesibilidad, hicieron posible las exportaciones en el sector de artesanías, exportaciones del sector textil y confecciones con efectos alentadores en ese sector productivo como queda demostrada en los informes sobre el particular.

Con respecto a la hipótesis específica 3, en aplicación y uso de los productos Marca Perú fundado en la satisfacción de los consumidores, el mejoramiento de las exportaciones de productos con valor agregado fue posible por el impulso y la aplicación correcta de las políticas de integración comercial iniciado por efecto del Decreto Legislativo $\mathrm{N}^{\circ} 668$, el ingreso del Perú al APEC, las firmas de los TLCs con los Estados Unidos, con Italia, la Alianza del Pacífico y en la formación y participación de Perú en los bloques económicos.

En cuanto a la hipótesis específica 4, en las estrategias de posicionamiento desarrolladas por la Marca Perú basadas en el estilo de vida de los consumidores, la preferencia por los productos saludables, se sustentó en la tendencia del mercado mundial a lo saludable, evidenciando una tendencia a favor del consumo de alimentos saludables, permitiendo así su conservación y entrega final al poseer el país como menciona la Asociación de Exportadores - ADEX una moderna infraestructura instalada, un desarrollo logístico portuario y aeroportuario, fuertes instituciones financieros, el asesoramiento 
adecuado por las Agencias de Aduanas y el mejoramiento de técnicas de rotulado, envase y embalaje de los productos exportables.

Con relación a la hipótesis específica 5, en el desarrollo de las estrategias de posicionamiento basadas en el producto Marca Perú, por sus ventajas frente a los otros de su misma especie, por ser una mejor alternativa y por poseer mayores grados de aceptación de los clientes se lograron la generación de más puestos de trabajo que permiten el incremento de la Población Económicamente Activa (PEA), produciendo un gran impacto en el desarrollo económico social del Perú.

La investigación realizada a través del trabajo de campo, se efectuó en las instituciones dedicadas a la promoción de productos de la Marca Perú, que tienen por objetivo crear una conciencia preferente de los diversos productos nacionales en los consumidores de otras naciones, proponiéndose objetivos que permitan su incremento cada año, los que dieron valor a la teoría sustentada en la variable independiente; $y$, con respecto a las exportaciones de los productos con valor agregado, en las empresas dedicadas a las exportaciones, sobre todo, aquellas que tienen por interés el mercado italiano, que dieron sustento a la teoría sustentada en la variable dependiente.

\section{CONCLUSIONES}

Las estrategias en el posicionamiento de la Marca Perú en el mercado italiano basadas en las características, la calidad del producto, en su uso y aplicación, en el estilo de vida de los consumidores y las competencias de dichos productos, mejoraron significativamente las exportaciones con valor agregado en los sectores químico metalmecánico, manufacturero, sustentadas en políticas de integración adecuadas y en la infraestructura portuaria, vial produciendo un incremento de la PEA y produciendo un desarrollo económico-social progresivo en el año 2018.

Se recomienda a las autoridades públicas y privadas del sector productivo regional, realizar estudios de mercado orientados a la actividad exportadora de productos nativos de la región, realizando capacitaciones y orientación en cada una de las actividades del sector.

Se recomienda a todas las autoridades educativas regionales, desarrollar centros de investigación para realizar un registro de productos exportables, investigando sus características para hacer posible su producción, mejoramiento y transformación en productos acabados.

Se recomienda a las autoridades públicas del Sector Industria y Turismo, hacer un registro de productos tanto tradicionales como nativos que permitan ser potencialmente exportables; entre ellos la palma datilera, palmera de los climas cálidos, por existir un mercado de necesidades insatisfechas con respecto a dicho producto puesto que existen en la región, las condiciones para su producción y convertirla en un producto acabado para su exportación.

\section{REFERENCIAS}

Ávalo Ortega, J. A., Yagüe Blanco, J. L., \& Cangahuala, G. (2016). El capital social y la planificación adaptativa en una comunidad industrial innovadora del Perú. Estudios Gerenciales, 32(139), 162-169. https://doi.org/10.1016/j.estger.2016.05.001

de Azambuja, G. M. M. P., García-Arrizabalaga, I., \& Rodríguez-Peña, G. (2019). Analyzing the purchase intention of Peruvian maca. Journal of Enterprising Communities: People and Places in the Global Economy. https://doi.org/10.1108/JEC-09-2019-0083

Bassols, N. (2016). Branding and promoting a country amidst a long-term conflict: The case of Colombia. Journal of Destination Marketing \& Management, 5(4), 314-324. https://doi.org/10.1016/j.jdmm.2016.10.001

BCRP (2018). 89. Producción manufacturera (variaciones porcentuales). (Año base 2007). BANCO CENTRAL DE RESERVA DEL PERÚ. Nota Semanal N ${ }^{\circ}$ 16-2018 (3 de mayo de 2018) Formato XLSX. Recuperado el 03-05-2018. 
http://www.bcrp.gob.pe/publicaciones/notasemanal/cuadros-estadisticos.html

Bruwer, J., Buller, C., Saliba, A.J. and Li, E. (2014), "Country-of-origin (COO) brand loyalty and related consumer behaviour in the Japanese wine market", International Journal of Wine Business Research, Vol. 26 No. 2, pp. 97-119

Curzi, D., Schuster, M., Maertens, M., \& Olper, A. (2020). Standards, trade margins and product quality: Firm-level evidence from Peru. Food Policy, 91, 101834. https://doi.org/10.1016/j.foodpol.2020.101834

Felipa, P. B. (2014). El desarrollo de la marca país: base para posicionarse a través de las exportaciones no tradicionales. Revista finanzas y política económica, 6(1), 115-140.

Grohs, R., Raies, K., Koll, O., \& Mühlbacher, H. (2016). One pie, many recipes: Alternative paths to high brand strength. Journal of Business Research, 69(6), 2244-2251. https://doi.org/10.1016/j.jbusres.2015.12.037

Lámbarry Vilchis, F. (2016). Structural analysis of the economic network of exports and imports of the Pacific Alliance and the Southern Common Market. Estudios Gerenciales, 32(141), 319-325. https://doi.org/10.1016/j.estger.2016.10.004

MINCETUR-OGEE-OEEI (2014). Indicadores Macroeconómicos 2015: Italia - Perú. Recuperado el 27-05-2018 de: http://www.acuerdoscomerciales.gob.pe/images/ stories/Reporte_bilateral/europa/RCB-I_TRI2015/italia_1_tri_15.pdf

MINCETUR (2017). Acuerdo Comercial entre Perú y la Unión Europea. Gobierno del Perú. Recuperado el 05-06-2018 de: http://www.acuerdoscomerciales.gob.pe/index.p hp?option=com_content $\&$ view=category\&layout $=$ blog $\& \mathrm{id}=50 \&$ Itemid $=73$

Niembro, A. (2017). Una tipología de empresas latinoamericanas exportadoras de servicios intensivos en conocimiento y los determinantes de su competitividad internacional. Estudios
Gerenciales, $\quad 33(142)$,

https://doi.org/10.1016/j.estger.2016.12.004

64-75.

Orihuela, J. C., \& Echenique, V. G. (2019). Volatile and spatially varied: the geographically differentiated economic outcomes of resourcebased development in Peru, 2001-2015. The Extractive Industries and Society, 6(4), 11431155. https://doi.org/10.1016/j.exis.2019.05.019

Pérez, J. A. H., Geldes, C., Kunc, M. H., \& Flores, A. (2019). New approach to the innovation process in emerging economies: The manufacturing sector case in Chile and Peru. Technovation, 79, 35-55. https://doi.org/10.1016/j.technovation.2018.02.0 12

Pino, C., Felzensztein, C., Zwerg-Villegas, A. M., \& Arias-Bolzmann, L. (2016). Non-technological innovations: Market performance of exporting firms in South America. Journal of Business Research, 69(10), 4385-4393. https://doi.org/10.1016/j.jbusres.2016.03.061

PROMPERÚ (2018). Guía de Mercado Multisectorial Italia. Recuperado el 29-05-2018 de: https://studylib.es/doc/4769899/italia---siicex

Schwarz, J., Mathijs, E., \& Maertens, M. (2019). A dynamic view on agricultural trade patterns and virtual water flows in Peru. Science of The Total Environment, 683, 719-728. https://doi.org/10.1016/j.scitotenv.2019.05.118

Silverman, H. (2015). Branding Peru: Cultural heritage and popular culture in the marketing strategy of PromPerú. In Encounters with Popular Pasts (pp. 131-148). Springer, Cham. https://doi.org/10.1007/978-3-319-13183-2_8

Tosoni, G. A. (2017). Tratados de libre comercio, crecimiento y producto potencial en Chile, México y Perú. ECONOMÍA unam, 14(42), 2446. https://doi.org/10.1016/j.eunam.2017.09.001

Vázquez López, R., \& Morales López, R. A. (2017). Diversificación de las exportaciones y competitividad externa en la industria. Hacia la 
construcción de una tipología para el caso de países de ingresos medios. Cuadernos de Economía, 40(114), 208-222.

https://doi.org/10.1016/j.cesjef.2016.09.002 